\title{
Omissão de macronutrientes no desenvolvimento de mudas de mogno africano $^{1}$
}

\author{
Cristiane Ramos Vieira²; Oscarlina Lúcia dos Santos Weber³; José Fernando Scaramuzza ${ }^{4}$
}

\begin{abstract}
Resumo: Objetivou-se identificar a sintomatologia visual das carências de macronutrientes, o crescimento e, os nutrientes mais absorvidos pelas mudas de mogno africano (Khaya anthotheca Welv.), submetidas à omissão de macronutrientes. As mudas foram produzidas em tubetes contendo areia, em seguida, transplantadas para tubos de PVC com areia e, submetidas por 15 dias à solução completa em nutrientes. Logo após, aplicou-se as soluções nutritivas, completa (macro e micronutrientes) e com omissão de N, P, K, Ca, $\mathrm{Mg}$ e S, por 90 dias, constituindo um delineamento inteiramente casualizado com sete tratamentos e quatro repetições. Os sintomas de deficiências foram descritos a cada cinco dias. Ao final do experimento, as mudas foram avaliadas em altura, diâmetro, biomassa seca, índice de qualidade de Dickson e concentração de macronutrientes. Nesse contexto, $\mathrm{N}, \mathrm{K}$ e S foram os macronutrientes que mais limitaram o crescimento e a qualidade das mudas de Khaya anthotheca.
\end{abstract}

Palavras - chave: Khaya anthotheca; Solução nutritiva; Elemento faltante; Nutrição de plantas.

\section{Macronutrients omission in development of african mahogany seedlings}

\begin{abstract}
The study aimed to identify the visual symptoms of macronutrients deficiencies, growth and the most absorbed by african mahogany (Khaya anthotheca Welv.) seedlings, submitted to macronutrients omission. The seedlings were grown in tubes containing sand, then were transplanted to PVC tube with sand and submitted for 15 days to complete nutrient solution. Then, were submitted for 90 days to complete nutrient solutions (macro and micronutrients) and omission of $\mathrm{N}, \mathrm{P}, \mathrm{K}, \mathrm{Ca}, \mathrm{Mg}$ and $\mathrm{S}$, constituting a randomized design with seven treatments and four replications. The symptoms of deficiency were described each five days. At the end of the experiment, the seedlings were evaluated to height, diameter, dry biomass, Dickson quality index and concentration of macronutrients. In this context, the growth and quality of Khaya anthotheca was more limited by $\mathrm{N}, \mathrm{K}$ and $\mathrm{S}$ omissions that were the most absorbed by the seedlings on complete solutions.
\end{abstract}

Keywords: Khaya anthotheca; Nutritive solution; Missing element; Plants nutrition.

\footnotetext{
${ }^{1}$ Recebido em 13.11.2014 e aceito para publicação como artigo científico em: 28.01.2015.

${ }^{2}$ Engenheira Florestal, Mestre em Ciências Florestais e Ambientais, Doutoranda em Agricultura Tropical pela Universidade Federal de Mato Grosso - Faculdade de Agronomia, Medicina Veterinária e Zootecnica. Av. Fernando Corrêa da Costa, $\mathrm{n}^{\circ} 2367$ - Bairro Boa Esperança. Cuiabá - MT - 78060-900. E-mail: <cris00986@hotmail.com>.

${ }^{3}$ Engenheira Agrônoma D.Sc., Professora do Departamento de Solos e Engenharia Rural da Universidade Federal de Mato Grosso. Av. Fernando Corrêa da Costa, nº 2367 - Bairro Boa Esperança. Cuiabá - MT - 78060-900. E-mail: <oscsan@uol.com.br>.

${ }^{4}$ Engenheiro Agrônomo D.Sc., Professor do Departamento de Solos e Engenharia Rural da Universidade Federal de Mato Grosso. Av. Fernando Corrêa da Costa, nº 2367 - Bairro Boa Esperança. Cuiabá - MT - 78060-900. E-mail: <jscaramuzza@uol.com.br>.
} 


\section{Introdução}

Os solos sob Cerrado são, em geral, de elevada acidez, altos teores de Al trocável, baixa disponibilidade de cátions básicos como $\mathrm{Ca} \mathrm{e}$ $\mathrm{Mg}$, e de $\mathrm{P}$ na forma disponível para as plantas, além dos micronutrientes $\mathrm{B}$ e $\mathrm{Cu}$. Características que comprometem o crescimento das plantas e, consequentemente, a produtividade das culturas. Práticas silviculturais como a calagem e a adubação com NPK são indispensáveis quando se trata de plantios em área de Cerrado.

De acordo com Silva et al. (2008) e Favare et al. (2012) o uso de corretivos e fertilizantes é fundamental na formação de mudas, o que, segundo Carlos et al. (2014) promove efeitos posteriores na melhora da produtividade, da qualidade e do estabelecimento dos plantios florestais. Para isso, o conhecimento das exigências nutricionais da espécie se faz necessário (FEITOSA et al., 2011). No entanto, como inexiste uma recomendação específica para cada espécie, a maioria das recomendações é baseada na estabelecida para o eucalipto, com algumas adaptações (CAIONE et al., 2012).

$A$ adubação inadequada facilita o ataque de pragas, promove o aparecimento de plantas com crescimento limitado, menor incremento médio anual, redução na taxa de formação da madeira e, consequentemente, na qualidade da mesma, desvalorizando-a. Ribeiro et al. (2006) sugerem que deve-se adicionar nutrientes ao solo conforme às exigências da planta. A determinação de doses adequadas e o relacionamento das mesmas com as respostas da planta aperfeiçoam a recomendação das quantidades de nutrientes a serem aplicadas ao substrato usado na produção das mudas (CRUZ et al., 2012). Nesse contexto, insere-se a Khaya anthotheca, cujo plantio em larga escala está sendo amplamente estimulado. Porém, pouco de sabe sobre seus requerimentos nutricionais.

Khaya anthotheca ou mogno africano é uma espécie da família Meliaceae, nativa do continente africano, com características semelhantes às do mogno brasileiro. Devido ao porte elevado, crescimento rápido e, características da madeira que favorecem a comercialização (fuste retilíneo, durabilidade, facilidade de trabalhar e a resistência ao ataque de pragas como a Hipsipyla grandella), é valorizado em todo o mundo. Essa demanda internacional torna a Khaya anthotheca uma alternativa para os silvicultores nacionais. Por isso, faz-se necessário desenvolver pesquisas que objetivam conhecer as exigências nutricionais dessa espécie.

A técnica da omissão de nutrientes é uma das mais utilizadas e mais rápidas para conhecer as exigências nutricionais de uma determinada espécie, segundo Malavolta (1980) serve para avaliar a fertilidade do solo podendo fornecer informações da necessidade de adubos, o que, segundo Benedetti et al. (2009) pode aprimorar a qualidade das mudas.

Venturin et al. (2005) observaram que a ausência de $\mathrm{P}$ e de $\mathrm{N}$ afetou drasticamente $\mathrm{o}$ crescimento de Eremanthus erythropappus, interferindo também, nos teores de $\mathrm{K}, \mathrm{Ca}, \mathrm{S}, \mathrm{B}$ e Zn. Vieira et al. (2008) concluíram que, N, P e $\mathrm{Mg}$ foram os macronutrientes que mais limitaram a produção de massa seca total em Moringa oleífera. Locatelli et al. (2007), Wallau et al. (2008), Matheus et al. (2011), Silva et al. (2011) e Andrade e Boaretto (2012) também realizaram estudos com omissões de nutrientes em Cedrela odorata, Swietenia macrophylla, Hymenaea courbaril var. stilbocarpa, Azadirachta indica e Schinus terebinthifolius, respectivamente.

O presente estudo teve por objetivo identificar a sintomatologia visual das carências de macronutrientes, avaliar o crescimento inicial e a qualidade das mudas de Khaya anthotheca, cultivadas em solução nutritiva.

\section{Material e métodos}

As mudas de Khaya anthotheca foram produzidas no viveiro florestal da Universidade Federal de Mato Grosso (UFMT), com sementes coletadas no município de Mocuba, Província da Zambézia, centro de Moçambique. Utilizou-se para isso, tubetes de $180 \mathrm{~cm}^{3}$ contendo areia, sendo uma semente por tubete. As primeiras 
germinações se deram oito dias após a semeadura (abril de 2010) e, após 20 dias, as mudas atingiram $20 \mathrm{~cm}$, estando aptas ao transplante.

As mudas foram transportadas para a casa de vegetação da Faculdade de Agronomia, Medicina Veterinária e Zootecnia (FAMEVZ) da UFMT e, transplantadas para tubos de PVC de $40 \mathrm{~cm}$ de comprimento, vedados com tela sobre recipiente plástico, preenchidos com areia previamente lavada e tratada com hipoclorito.

Após transplante, as mudas permaneceram em período de adaptação, com irrigação uma vez ao dia, com $50 \mathrm{~mL}$ de água em copo descartável, exceto no dia de aplicação da solução. Considerando-as adaptadas quando apresentando a formação de novas brotações, o que ocorreu 15 dias após o transplante.

Em seguida, as mudas passaram pela adaptação da solução nutritiva completa, garantindo condições nutritivas semelhantes em todos os indivíduos. Para isso, utilizou-se solução completa com $1 / 4$ de força, conforme recomendado por Sarruge (1975), por 15 dias, e renovação de solução a cada cinco dias. $\mathrm{O}$ pH da solução foi mantido a 5,9 $\pm 0,1$ e quando necessário foram feitas as correções com $\mathrm{HCl}$ 1,0 M ou $\mathrm{NaOH}$ 1,0 M.

Encerrado o período de adaptação às soluções nutritivas, as mudas foram submetidas, por 90 dias, às soluções completa e com elemento faltante, com $50 \mathrm{~mL}$ de solução com $100 \%$ de força, renovadas a cada cindo dias. Dispostas em delineamento inteiramente casualizado, com sete tratamentos e quatro repetições, totalizando 28 parcelas: $\mathrm{C}$ (solução nutritiva completa), $-\mathrm{N}$ (omissão de N), -P (omissão de P), -K (omissão de $\mathrm{K}$ ), -Ca (omissão de $\mathrm{Ca}$ ), -Mg (omissão de $\mathrm{Mg}$ ) e -S (omissão de $\mathrm{S}$ ). As soluções foram preparadas com reagentes puros (P.A.), sendo que, a solução completa, segundo Sarruge (1975) teve a seguinte composição: $\mathrm{N}-210$ $\mathrm{mg} / \mathrm{L} ; \mathrm{P}-31 \mathrm{mg} / \mathrm{L} ; \mathrm{K}-234 \mathrm{mg} / \mathrm{L} ; \mathrm{Ca}-200$ $\mathrm{mg} / \mathrm{L} ; \mathrm{Mg}-48 \mathrm{mg} / \mathrm{L} ; \mathrm{S}-64 \mathrm{mg} / \mathrm{L}$.

Nesse período, a irrigação se deu uma vez por dia com $50 \mathrm{~mL}$ de água, exceto no dia de aplicação das soluções nutritivas, em que se omitiu a irrigação.
As sintomatologias visuais de deficiências foram descritas a cada cinco dias durante 90 dias. Enquanto que, a caracterização morfológica foi realizada apenas ao final do experimento. A altura da parte aérea foi medida com régua graduada, a $5 \mathrm{~cm}$ da superfície do solo. O diâmetro foi medido com paquímetro digital, na região do coleto. Para a obtenção da biomassa seca, as mudas foram retiradas do tubo de PVC, seccionadas em parte aérea e parte radicular. As raízes foram lavadas e, em seguida, todo o material foi levado à estufa de circulação forçada de ar a $65^{\circ} \mathrm{C}$. Ao atingir peso constante, o material seco foi pesado em balança analítica com precisão de 0,0005g. Logo após, determinou-se o índice de qualidade de Dickson, conforme equação descrita em Dickson et al. (1960).

O material seco foi moído em moinho tipo Willey e, posteriormente, submetido às digestões sulfúrica e nitro-perclórica para determinações das concentrações de macronutrientes conforme metodologia proposta por Malavolta et al. (1997). A saber: $\mathrm{N}$ total por semi-micro Kjeldahl; $\mathrm{P}$ por colorimetria do metavanadato; S por turbidimetria do sulfato de bário; K por fotometria de chama de emissão; $\mathrm{Ca}$ e $\mathrm{Mg}$ por quelatometria com EDTA, obtidas por diferença.

Para o processamento e análise dos dados utilizou-se o software Assistat 7.6 beta (Silva e Azevedo, 2002), e a análise estatística foi realizada aplicando-se a técnica de análise de variância e comparações múltiplas de médias pelo teste Tukey considerando significância de $5 \%$.

\section{Resultados e Discussão}

Crescimento inicial de mudas de Khaya anthotheca

Não houve significância no crescimento em altura e formação de massa seca na parte radicular (Tabela 1). Isso pode ter ocorrido porque as mudas de Khaya anthotheca conseguiram absorver, no processo de 
adaptação, os nutrientes necessários para seu crescimento até o período de análise do estudo. No entanto, pode-se verificar que, a omissão de $\mathrm{N}$ promoveu queda de $37 \%$ no crescimento em altura e de $51,9 \%$ na biomassa radicular das mudas, comparado ao tratamento completo. Dessa forma, pode-se considerar que, $\mathrm{N}$ é o nutriente mais requerido pela espécie, como observado para Toona ciliata (MORETTi et al., 2011), Croton urucurana (SORREANO et al., 2011), Caesalpinia echinata (VALERI et al., 2014) e Khaya ivorensis Corcioli et al. (2014), dentre outros.

Tabela 1: Crescimento inicial das mudas de Khaya anthotheca sob omissão de macronutrientes.

Table 1: Initial growth of Khaya anthotheca seedlings in macronutrient omissions.

\begin{tabular}{cccccc}
\hline Tratamento & $\mathbf{H}^{*}(\mathbf{c m})$ & DC* $(\mathbf{m m})$ & BioPA $^{*}(\mathbf{g})$ & BioR $^{*}(\mathbf{g})$ & IQD $^{*}$ \\
\hline Completa & $61,67 \mathrm{a}$ & $7,16 \mathrm{ab}$ & $8,10 \mathrm{abc}$ & $2,39 \mathrm{a}$ & $0,87 \mathrm{ab}$ \\
$-\mathrm{N}$ & $38,67 \mathrm{a}$ & $5,28 \mathrm{~b}$ & $3,16 \mathrm{c}$ & $1,15 \mathrm{a}$ & $0,43 \mathrm{c}$ \\
$-\mathrm{P}$ & $54,33 \mathrm{a}$ & $7,97 \mathrm{a}$ & $8,68 \mathrm{ab}$ & $2,17 \mathrm{a}$ & $1,01 \mathrm{a}$ \\
$-\mathrm{K}$ & $47,33 \mathrm{a}$ & $5,56 \mathrm{ab}$ & $4,37 \mathrm{abc}$ & $1,36 \mathrm{a}$ & $0,49 \mathrm{c}$ \\
$-\mathrm{Ca}$ & $57,67 \mathrm{a}$ & $6,91 \mathrm{ab}$ & $6,19 \mathrm{abc}$ & $1,63 \mathrm{a}$ & $0,65 \mathrm{bc}$ \\
$-\mathrm{Mg}$ & $54,67 \mathrm{a}$ & $7,18 \mathrm{ab}$ & $8,95 \mathrm{a}$ & $2,24 \mathrm{a}$ & $0,97 \mathrm{ab}$ \\
$-\mathrm{S}$ & $45,67 \mathrm{a}$ & $5,73 \mathrm{ab}$ & $3,86 \mathrm{bc}$ & $1,43 \mathrm{a}$ & $0,50 \mathrm{c}$ \\
\hline $\mathbf{C V}(\boldsymbol{\%})$ & $\mathbf{1 6 , 1 6}$ & $\mathbf{1 3 , 3 0}$ & $\mathbf{2 8 , 7 9}$ & $\mathbf{2 5 , 4 3}$ & $\mathbf{1 7 , 1 2}$ \\
\hline
\end{tabular}

*Em que: H - altura; DC - diâmetro de colo; BioPA - biomassa da parte aérea; BioPR - biomassa parte radicular; IQD índice de qualidade de Dickson. Médias seguidas de mesma letra não diferem estatisticamente entre si pelo teste Tukey a 5\% de probabilidade.

A limitação no crescimento de mudas submetidas à omissão de $\mathrm{N}$ se deve, provavelmente, ao fato de o elemento ser exigido em grandes quantidades pelas plantas, segundo Raij (1991), além de fazer parte da estrutura de aminoácidos, proteínas, bases nitrogenadas, ácidos nucléicos, enzimas, pigmentos e produtos secundários, participa de processos como absorção iônica, fotossíntese, respiração, multiplicação e diferenciação celular (MARSCHNER, 1995; MALAVOLTA et al., 1997). Razão pela qual foi o nutriente cuja omissão mais interferiu negativamente no crescimento em diâmetro das mudas $(26,3 \%$ menor em relação ao tratamento completo). Enquanto que, na omissão de $\mathrm{P}$, o crescimento em diâmetro foi superior ao do tratamento completo, o que pode indicar a não exigência desse nutriente em grandes quantidades nessa fase de desenvolvimento. Segundo Sarcinelli et al. (2004), isso pode ocorrer também porque o período inicial de fornecimento de solução completa às plantas pode ter permitido $o$ acúmulo do nutriente de forma a estocá-lo e o mesmo passou a ser utilizado eficientemente pela planta quando da sua supressão. Além disso, Wallau et al. (2008) explicaram que, as sementes contêm uma reserva de $\mathrm{P}$ (P-fitina) que pode fornecer quantidade suficiente desse nutriente, na fase inicial de desenvolvimento das plantas.

O menor crescimento, provocado pela omissão de N, limitou a produção de biomassa na parte aérea $(61 \%$ menor que o tratamento completo). Resultados semelhantes aos observados por Moretti et al. (2011), Silva et al. (2011), Camacho et al. (2014) e Corcioli et al. (2014), com Toona ciliata, Azadirachta indica, Bombacopsis glabra e Khaya ivorensis, respectivamente. $\mathrm{O}$ que está relacionado ao papel desempenhado pelo $\mathrm{N}$ no crescimento das plantas. Além da omissão de N, a omissão de $\mathrm{S}$ também influenciou na produção de biomassa nas mudas $(52,3 \%$ menor que o tratamento completo), como observado por Barroso et al. (2005) e Silva et al. (2005). Isso pode ter ocorrido porque $\mathrm{S}$ é constituinte essencial das proteínas, sua deficiência resulta na inibição da síntese de proteínas e aminoácidos (MARSCHNER, 1995). Enquanto que, a 
omissão de $\mathrm{Mg}$ promoveu maior produção de biomassa na parte aérea, indicando que, este não é um dos macronutrientes mais limitantes do crescimento inicial das mudas da espécie.

Portanto, as mudas tiveram a qualidade significativamente inferior, provavelmente devido a baixa produção de massa seca, sendo N, $\mathrm{K}$ e $\mathrm{S}$ os macronutrientes mais requeridos pelas mudas de Khaya anthotheca em sua fase inicial de crescimento.

Sintomatologias visuais das deficiências de macronutrientes

\section{Deficiências de N}

Os primeiros sintomas de deficiência foram observados ao $62^{\circ}$ dia, com perda de coloração das folhas velhas (Figura 1). Marques et al. (2004) atribuíram esse fato à redução na síntese de clorofila, o que, segundo Sorreano et al. (2011) é resultado da proteólise e redistribuição. Em seguida, observou-se clorose na ponta de folhas velhas ou nas bordas, que evoluiu para necrose. Sintomas semelhantes aos observados por Wallau et al. (2008), Andrade e Boaretto (2011), Corcioli et al. (2014) e Moretti et al. (2014). Além de inserções foliares mortas ao longo do caule ou folhas atrofiadas. Resultados que, provavelmente, estão relacionados às funções do $\mathrm{N}$ como constituinte de muitos componentes da célula vegetal, incluindo aminoácidos e ácidos nucléicos, que estão ligados aos processos de divisão celular (TAIZ e ZEIGER, 2004).

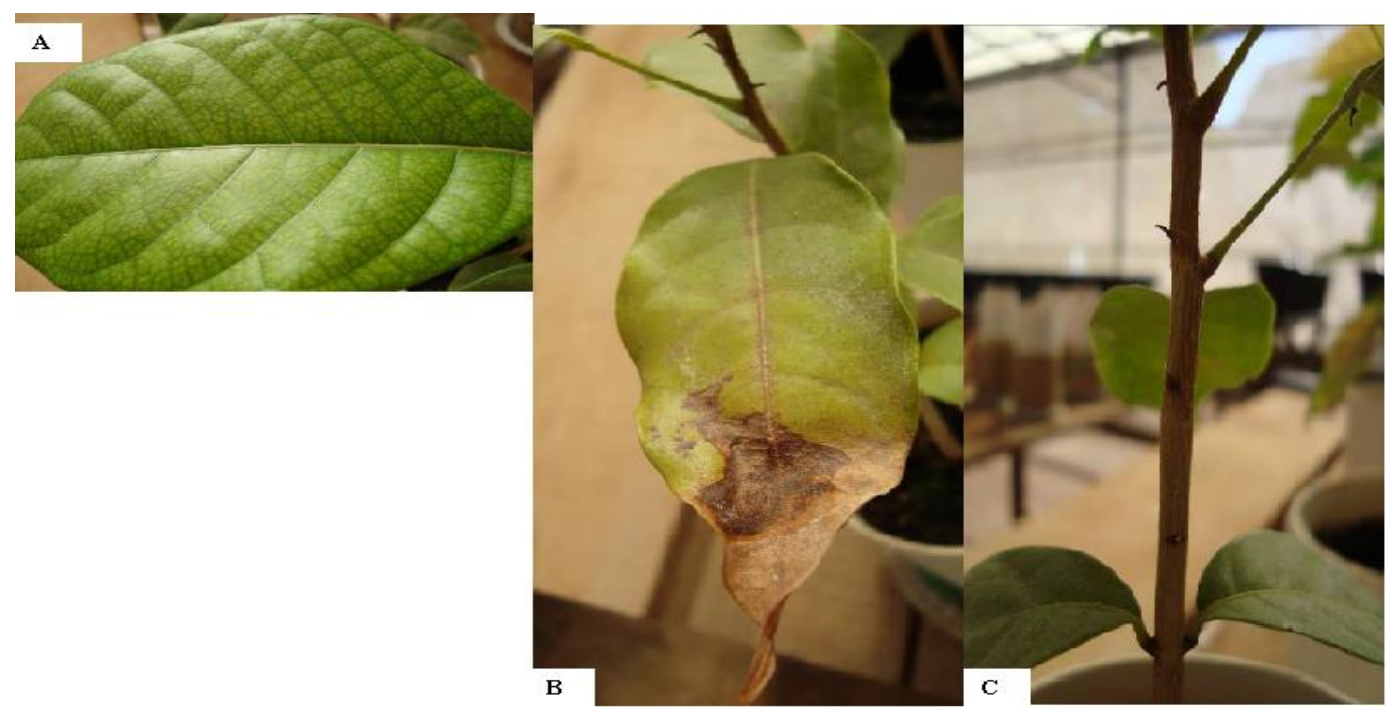

Figura 1: A: Folha nova com cor verde claro; B: clorose na ponta das folhas; C: novas brotações.

Figure 1: A: New leaf with light green color; B: chlorosis at the tip of the leaves; C: new growths.

\section{Deficiências de P}

Os primeiros sintomas (Figura 2) foram observados ao $62^{\circ}$ dia, quando as folhas velhas se tornaram mais claras, e algumas apresentaram clorose nas bordas. Observou-se ainda, queda de folhas velhas mesmo verdes e, novas inserções com folhas atrofiadas. Camacho et al. (2014) também observaram aumento no número de brotações com a omissão de P em Bombacopsis glabra.

\section{Deficiências de K}

Os primeiros sintomas (Figura 3) foram observados ao $30^{\circ}$ dia, quando a coloração das folhas velhas foi comprometida, progredindo para tom bastante claro. Observou-se também, folhas velhas com encarquilhamento para cima e começo de clorose na região do ápice ou com início pelas bordas. De acordo com Marschner (1995), isso ocorre devido o acúmulo de putrescina. Sintomas semelhantes foram 
observados por Sorreano et al. (2011) em mudas de Croton urucurana; por Valeri et al. (2014), em Caesalpinia echinata e; por Corcioli et al. (2014), em Khaya ivorensis. Algumas inserções apresentaram folhas mortas ao longo do caule ou com coloração clara, encarquilhamento para baixo e leve enrugamento. Wallau et al. (2008) também observaram mudas de Swietenia macrophylla com folhas encarquilhadas para baixo após omitir K.

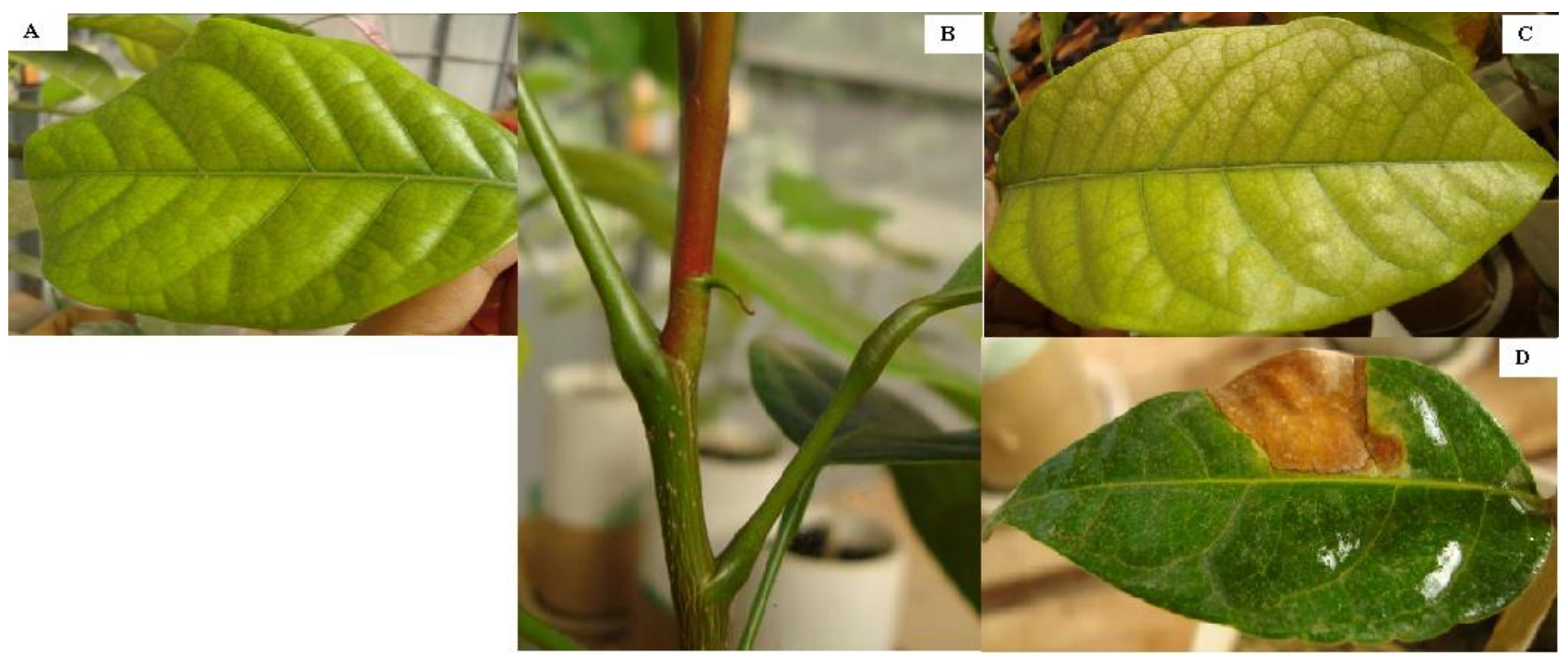

Figura 2: A: Folha com formato irregular; B: novas inserções com folhas atrofiadas; C: folhas velhas mais claras; D - clorose nas bordas.

Figure 2: A: Leaf with irregularly shaped; B: new inserts with atrophied leaves; C: clearer old leaves; D - chlorosis at the edges.

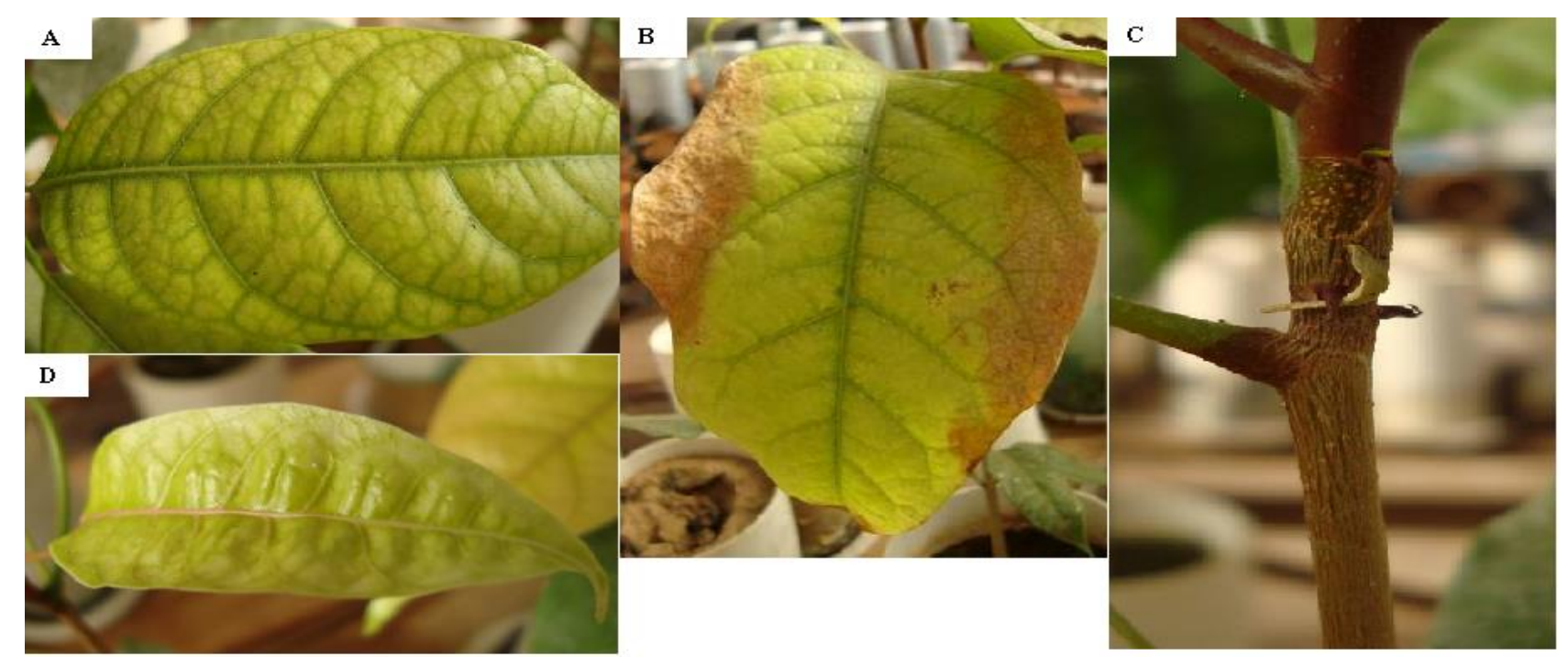

Figura 3: A: Folha verde claro; B: clorose nas bordas; C: inserções com folhas atrofiadas; D: folhas com ápice para baixo. Figure 3: A: Leaf light green; B: chlorosis at the edges; C: inserts with atrophied leaves; D: leaves with apex down.

Deficiências de Ca

Os primeiros sintomas (Figura 4) foram observados ao $68^{\circ}$ dia, com o aparecimento de folhas novas e velhas com coloração mais clara e bordas levemente onduladas. Observou-se clorose nas bordas das folhas novas, também descrita por Wallau et al. (2008) e Moretti et al. (2011); sendo que algumas apresentaram atrofiamento no formato, também observado por 
Sorreano et al. (2011) e ainda; novas inserções com folhas mortas. Isso porque os sintomas de crescimento tanto da parte aérea quanto da deficiências de $\mathrm{Ca}$ ocorrem nos pontos de

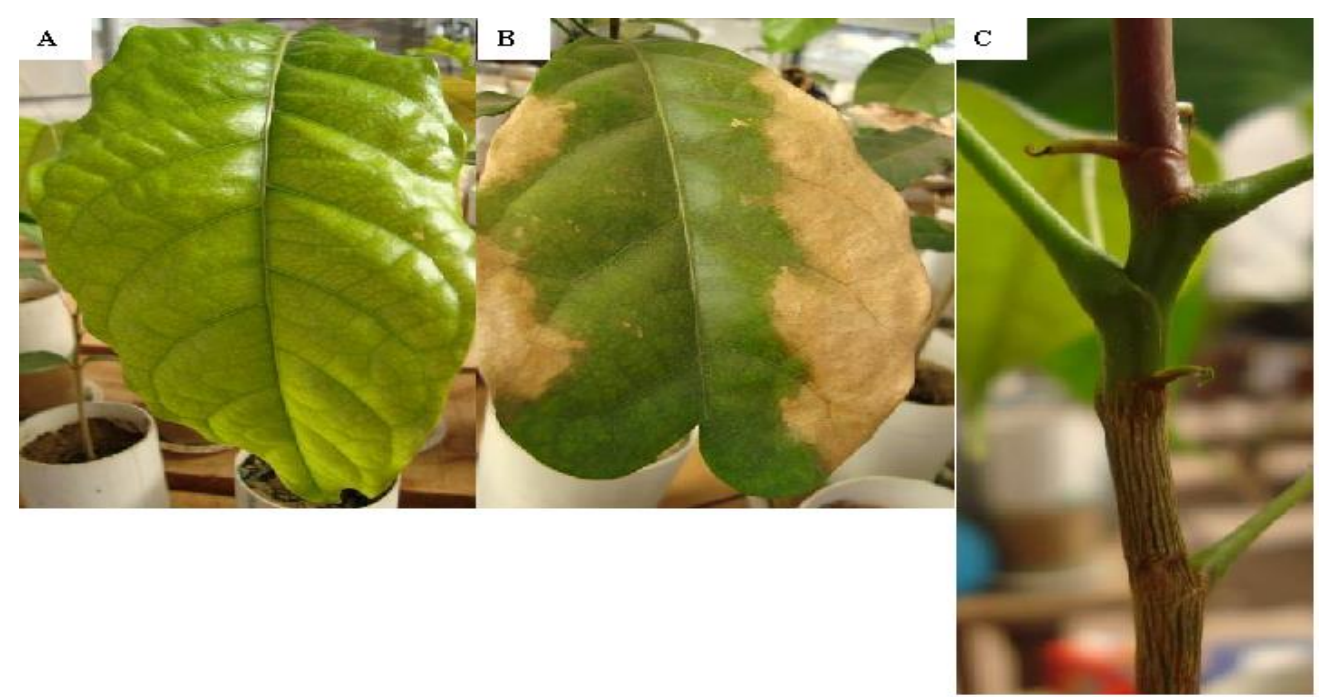

Figura 4: A: Folha com borda ondulada; B: clorose nas bordas; C: inserções com folhas atrofiadas.

Figure 4: A: Leaf with wavy edge; B: chlorosis at the edges; C: inserts with atrophied leaves

Deficiências de $\mathrm{Mg}$

Os primeiros sintomas (Figura 5) foram observados ao $35^{\circ}$, com o aparecimento de folhas novas mais claras. A despigmentação é característica determinante dos efeitos da deficiência de $\mathrm{Mg}$, pois o elemento é parte da estrutura da molécula de clorofila (TAIZ e ZEIGER, 2004). Esse sintoma também foi observado em folhas velhas, porém, com aspecto ondulado nas bordas; em outras, observou-se clorose, também descrita por Wallau et al. (2008), Sorreano et al. (2011), Camacho et al. (2014) e Valeri et al. (2014). Segundo Malavolta et al. (1997) e Taiz e Zeiger (2004), a clorose aparece em decorrência da redução no teor de clorofila. No entanto, a clorose progrediu para necrose nas bordas e/ou na ponta das folhas, provocando o seu encarquilhamento para cima, além de inserção com folhas atrofiadas.
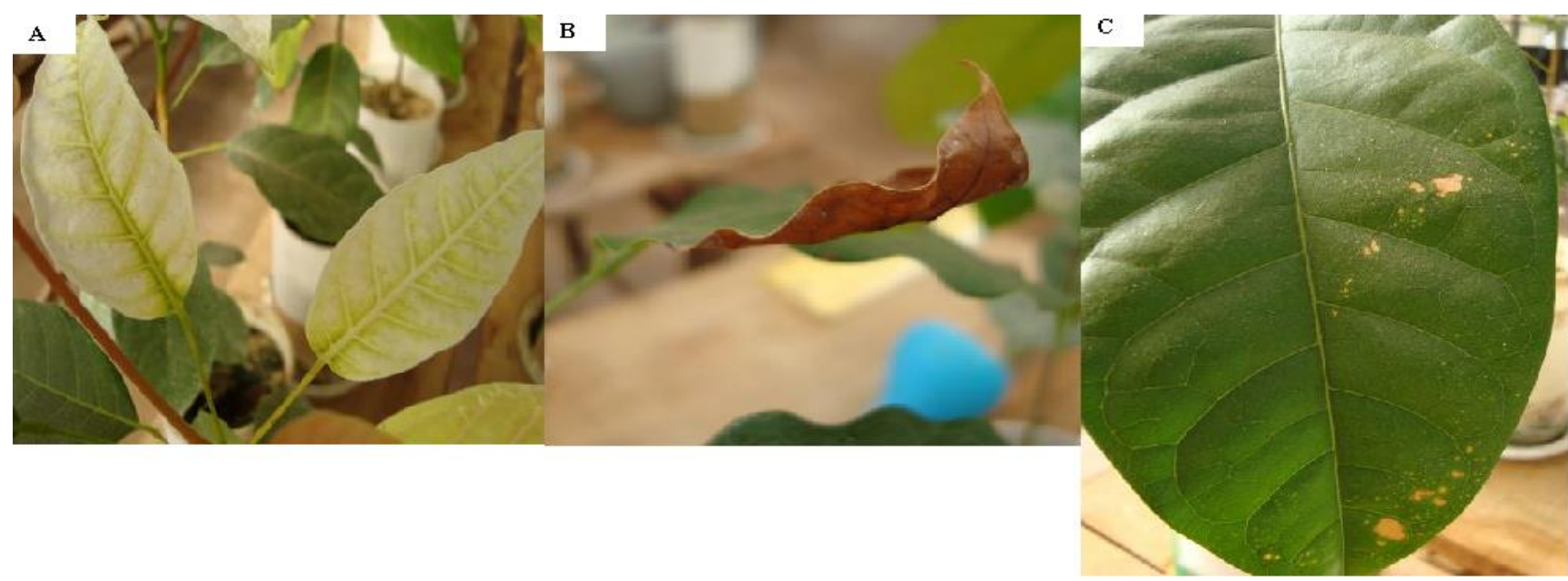

Figura 5: A: Folhas mais claras; B: folhas com bordas onduladas; C: clorose.

Figure 5: A: Leaf lighter; B: leaves with wavy edges; C: chlorosis. 
Deficiências de S

Os primeiros sintomas (Figura 6) foram observados ao $35^{\circ}$ dia, com a perda de coloração das folhas velhas, que se tornaram deformadas e com pontas atrofiadas ou encarquilhadas para baixo. Algumas apresentaram ainda, clorose começando pelas bordas, sintoma também observado por Wallau et al. (2008) e Camacho et al. (2014) em mudas de Swietenia macrophylla e de Bombacopsis glabra, respectivamente. Algumas folhas novas se tornaram verde claro, progredindo para total perda de coloração. As mudas apresentaram ainda, excesso de brotações com folhas atrofiadas.

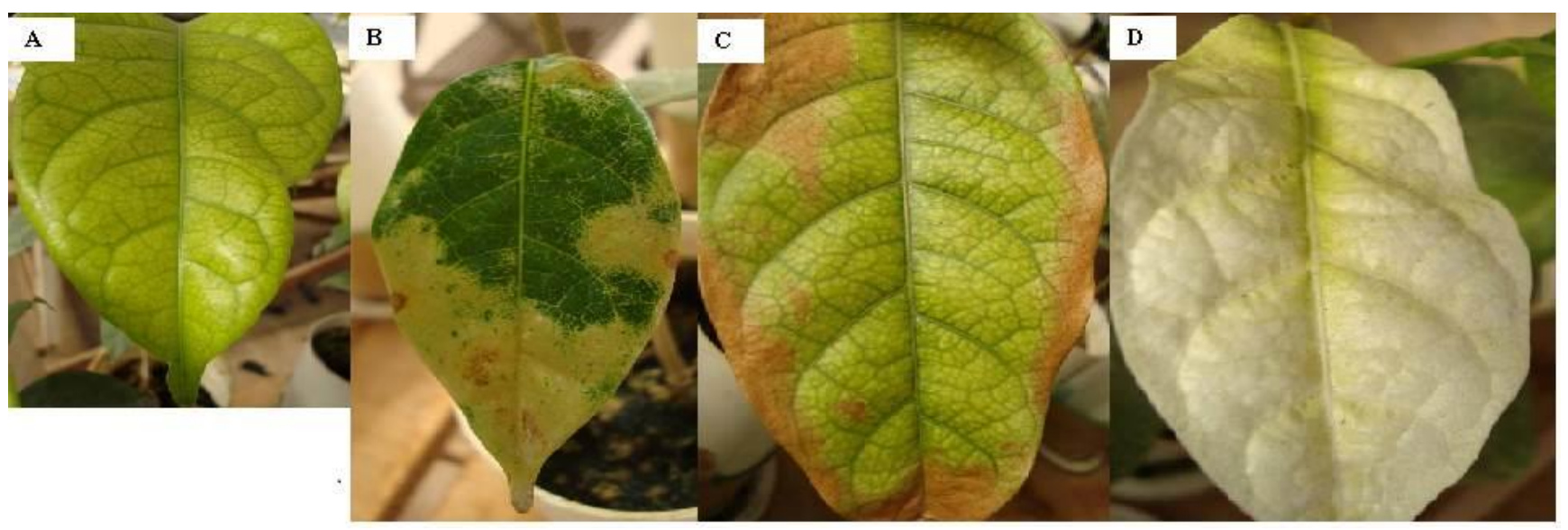

Figura 6: A: Folha formato irregular; B e C: clorose; D: folhas claras.

Figure 6: A: Leaf with irregular shaped; B and C: chlorosis; D: lighter leaves.

Concentrações de macronutrientes

As concentrações dos macronutrientes na parte aérea das mudas de Khaya anthotheca estão apresentadas na Tabela 2.

Tabela 2: Concentrações de macronutrientes, em $\mathrm{g} \mathrm{kg}^{-1}$, na parte aérea de mudas de Khaya anthotheca submetidas às omissões de macronutrientes.

Table 2: Macronutrients concentration, in $\mathrm{g} \mathrm{kg}^{-1}$, in aerial parts of Khaya anthotheca seedlings submitted to omissions of macronutrients.

\begin{tabular}{ccccccc}
\hline Tratamento & $\mathbf{N}$ & $\mathbf{P}$ & $\mathbf{K}$ & $\mathbf{C a}$ & $\mathbf{M g}$ & $\mathbf{S}$ \\
\hline Completo & $19,69 \mathrm{a}$ & $6,63 \mathrm{a}$ & $6,76 \mathrm{a}$ & $1,28 \mathrm{a}$ & $1,85 \mathrm{a}$ & $8,11 \mathrm{a}$ \\
$-\mathrm{N}$ & $10,45 \mathrm{c}$ & $6,47 \mathrm{ab}$ & $6,25 \mathrm{ab}$ & $0,96 \mathrm{a}$ & $2,01 \mathrm{a}$ & $2,34 \mathrm{c}$ \\
$-\mathrm{P}$ & $14,75 \mathrm{~b}$ & $3,28 \mathrm{c}$ & $5,67 \mathrm{ab}$ & $1,17 \mathrm{a}$ & $2,30 \mathrm{a}$ & $1,12 \mathrm{c}$ \\
$-\mathrm{K}$ & $19,13 \mathrm{a}$ & $4,76 \mathrm{abc}$ & $5,08 \mathrm{~b}$ & $1,38 \mathrm{a}$ & $2,17 \mathrm{a}$ & $2,73 \mathrm{bc}$ \\
$-\mathrm{Ca}$ & $15,12 \mathrm{~b}$ & $5,33 \mathrm{ab}$ & $5,75 \mathrm{ab}$ & $1,07 \mathrm{a}$ & $2,05 \mathrm{a}$ & $5,44 \mathrm{ab}$ \\
$-\mathrm{Mg}$ & $17,64 \mathrm{ab}$ & $4,58 \mathrm{bc}$ & $6,33 \mathrm{ab}$ & $1,38 \mathrm{a}$ & $2,17 \mathrm{a}$ & $1,87 \mathrm{c}$ \\
$-\mathrm{S}$ & $19,60 \mathrm{a}$ & $6,46 \mathrm{ab}$ & $6,75 \mathrm{a}$ & $1,49 \mathrm{a}$ & $2,43 \mathrm{a}$ & $3,70 \mathrm{bc}$ \\
\hline CV $(\%)$ & 8,86 & 13,58 & 8,92 & 23,04 & 30,12 & 30 \\
\hline
\end{tabular}

Médias seguidas de mesma letra não diferem estatisticamente entre si pelo teste de Tukey a 5\% de probabilidade. 
As maiores concentrações de $\mathrm{N}$ foram encontradas nos tratamentos completo, $-\mathrm{K}$ e $-\mathrm{S}$, com 19,69 $\mathrm{g} \mathrm{kg}^{-1}, 19,13 \mathrm{~g} \mathrm{~kg}^{-1} \mathrm{e}$ 19,60 $\mathrm{g} \mathrm{kg}^{-1}$, respectivamente. Valores semelhantes aos obtidos por Souza et al. (2010) em Swietenia macrophylla, Moretti et al. (2011) em Toona ciliata e Valeri et al. (2014) em Caesalpinia echinata. Wallau et al. (2008) também observaram aumento na concentração de $\mathrm{N}$ devido a omissão de K em mudas de Swietenia macrophylla. Segundo Souza et al. (2010) devido a competição entre $\mathrm{N}$ e K. Isso porque, $\mathrm{K}$ na solução do solo encontra-se na forma iônica $\mathrm{K}^{+}$, sendo assim, concentrações elevadas de $\mathrm{NH}^{4+}$ reduzem a absorção do $\mathrm{K}$ por inibição competitiva (MALAVOLTA, 1980). De acordo com Zhang et al. (2010) o antagonismo entre eles pode ser atribuído como simples efeito competitivo, no mesmo sítio de absorção. Considerando a recomendação de Malavolta et al. (1997) para os quais as concentrações adequadas de $\mathrm{N}$ devem variar de 12 a $35 \mathrm{~g} \mathrm{~kg}^{-1}$, somente em mudas submetidas à omissão de $\mathrm{N}$ não se observou concentrações dentro da faixa ideal, sendo $47 \%$ inferiores às do tratamento completo. Resultado que explica a redução no crescimento das mudas de Khaya anthotheca.

As maiores concentrações de $\mathrm{P}$ foram encontradas no tratamento completo e as menores na omissão de P. No entanto ao comparar com o tratamento completo, as omissões de $\mathrm{N}, \mathrm{K}, \mathrm{Ca}, \mathrm{Mg}$ e $\mathrm{S}$ também reduziram as concentrações de P. Considerando que, para Malavolta et al. (1997) as concentrações foliares de $\mathrm{P}$ devem estar entre 1,0 a 2,3 $\mathrm{g} \mathrm{kg}^{-1}$, todos os tratamentos proporcionaram concentrações acima da ideal. O que pode ter contribuído para o crescimento das mudas. Sendo os valores obtidos semelhantes aos observados por Sorreano et al. (2011) em Croton urucurana, por Vieira et al. (2011) em Amburana acreana e por Valeri et al. (2014) em Caesalpinia echinata.

As menores concentrações de $\mathrm{K}$ foram verificadas na omissão do mesmo nutriente, assim como observado por Wallau et al. (2008) em mudas de Switenia macrophylla. Enquanto que, a omissão de $\mathrm{S}$ aumentou a concentração de $\mathrm{K}$, permanecendo igual à do tratamento completo. De acordo com Mendonça et al. (1999), as concentrações adequadas de K nas folhas de Myracrodruon urundeuva com 120 dias, variaram entre 4 e $18,5 \mathrm{~g} \mathrm{~kg}^{-1}$, o que ocorreu em todos os tratamentos testados em Khaya anthotheca. Porém, segundo Malavolta et al (1997) concentrações foliares de K para espécies florestais (em geral) devem estar entre 10 a $15 \mathrm{~g}$ $\mathrm{kg}^{-1}$, nesse caso, nenhum tratamento atingiu a faixa ideal. Com valores semelhantes aos observados por Vieira et al. (2011) em Amburana acreana e por Carlos et al. (2014) em Caryocar brasiliense. Contrariamente ao que comumente é observada na literatura, a omissão de $\mathrm{Ca}$ reduziu as concentrações de $\mathrm{K}$. Isso ocorre, segundo Malavolta et al. (1997), porque o Ca em concentração não muito elevada no substrato apresenta relação de sinergismo com $\mathrm{K}$.

Não houve diferença significativa na concentração de $\mathrm{Ca}$ nas mudas de Khaya anthotheca. Segundo Malavolta et al. (1997), concentrações foliares de $\mathrm{Ca}$ estão adequadas quando entre 3,0 a 12,0 $\mathrm{g} \mathrm{kg}^{-1}$. Assim, todos os tratamentos apresentaram concentrações abaixo da ideal, provavelmente, devido à quantidade de Ca presente nas soluções nutritivas.

Não se observou diferença significativa na concentração de $\mathrm{Mg}$ nas mudas de Khaya anthotheca. Malavolta et al. (1997) sugeriram, para $\mathrm{Mg}$, uma faixa adequada de 1,5 a 5,0 $\mathrm{g} \mathrm{kg}^{-}$ 1 , portanto, todos os tratamentos apresentaram concentrações adequadas, com valores semelhantes às observadas por Carlos et al. (2014) em mudas de Caryocar brasiliense e por Valeri et al. (2014) em mudas de Caesalpinia echinata. $\mathrm{O}$ adequado crescimento das mudas de Khaya anthotheca em omissão de $\mathrm{Mg}$ pode ser explicado pela quantidade de $\mathrm{Mg}$ nas soluções nutritivas, que pode estar adequada para a espécie estudada, ou ainda, ao baixo requerimento das mudas dessa espécie na fase inicial de crescimento.

Observou-se que as mudas do tratamento completo apresentaram as maiores concentrações de $\mathrm{S}$. Enquanto que, as omissões de N, P, K, Ca, Mg e S reduziram a concentração de S. Para Malavolta et al. (1997), as 
concentrações de $\mathrm{S}$ devem estar entre 1,4 a 2,0 g $\mathrm{kg}^{-1}$. No entanto, nos tratamentos completo, $-\mathrm{N}$, $-\mathrm{K}$, -Ca e -S estiveram acima da ideal; na omissão de $\mathrm{P}$, abaixo e; na omissão de $\mathrm{Mg}$ permaneceram na faixa recomendada. Wallau et al. (2008) também observaram concentrações de $\mathrm{S}$ acima da ideal em mudas de Swietenia macrophylla. Porém, as concentrações foram semelhantes às observadas por Valeri et al. (2014) em mudas de Caesalpinia echinata. O que pode ter ocorrido devido às concentrações do elemento nas soluções nutritivas e à possibilidade de absorção do $\mathrm{S}$ atmosférico. De acordo com Freedman (1995) $\mathrm{SO}_{2}$ pode ser absorvido pelos estômatos. Nesse caso, Pedroso (2007) relata que, o $\mathrm{SO}_{2}$ é dissolvido em água, gerando dois compostos, o sulfeto de hidrogênio e o sulfito, estes compostos são então, fotooxidados em sulfato, um composto menos tóxico para a planta.

Portanto, as quantidades de nutrientes utilizadas na preparação das soluções nutritivas podem não ser as ideias para a espécie Khaya anthotheca, devendo-se, assim, desenvolver pesquisas para estudar as quantidades necessárias para a mesma.

Considerando as concentrações nas mudas de Khaya anthotheca em solução completa, o requerimento da espécie em sua fase inicial de crescimento foi: $\mathrm{N}>\mathrm{S}>\mathrm{K}>\mathrm{P}>\mathrm{Mg}>\mathrm{Ca}$.

\section{Conclusões}

Os sintomas de deficiências de macronutrientes em mudas de Khaya anthotheca são condizentes aos observados na literatura e, podem ser facilmente descritos.

$\mathrm{O}$ crescimento de mudas de Khaya anthotheca foi limitado, principalmente, pelas ausências de $\mathrm{N}, \mathrm{K}$ e $\mathrm{S}$, sendo essas também, os que mais limitaram a qualidade das mesmas.

\section{Referências}

ABRAF ANDRADE, M. L. F.; BOARETTO, A. E. Deficiência nutricional em plantas jovens de aroeira-pimenteira (Schinus terebinthifolius Raddi). Scientia Forestalis, v. 40, n. 95, p. 383392, 2012.

BARROSO, D. G. et al. Diagnóstico de deficiências de macronutrientes em mudas de teca. Revista Árvore, v. 29, n. 5, p. 671-679, 2005.

BENEDETTI, E. L. et al. Crescimento e sintomas em mudas de espinheira-santa com omissão de nitrogênio, fósforo e potássio. Floresta, v. 39, n. 2, p. 335-343, 2009.

CAIONE, G.; LANGE, A.; SCHONINGER, E.L. Crescimento de mudas de Schizolobium amazonicum (Huber ex Ducke) em substrato fertilizado com nitrogênio, fósforo e potássio. Scientia Forestalis, v. 40, n. 94, p. 213-221, 2012.

CAMACHO, M. A.; CAMARA, A. P.; ZARDIN, A. R. Diagnose visual de deficiência de nutrientes em mudas de Bombacopsis glabra. Cerne, v. 20, n. 3, p. 427-431, 2014.

CARLOS, L. et al. Crescimento e nutrição mineral de mudas de pequi sob efeito da omissão de nutrientes. Ciência Florestal, v. 24, n. 1, p. 13-21, 2014.

CORCIOLI, G.; BORGES, J. D.; JESUS, R. P. Sintomas de deficiência nutricional de macronutrientes em mudas de Khaya ivorensis cultivadas em solução nutritiva. Pesquisa Florestal Brasileira, v. 34, n. 78, p. 159-164, 2014.

CRUZ, C. A. F. et al. Produção de mudas de canafístula cultivadas em Latossolo vermelho amarelo álico em resposta a macronutrientes. Cerne, v. 18, n. 1, p. 87-98, 2012.

DICKSON, A.; LEAF, A. L.; HOSNER, J. F. Quality appraisal of white spruce and white pine seedlings stock in nurseries. Forest Chronicle, v. 36, p. 10-13, 1960. 
FAVARE, L. G.; GUERRINI, I. A.; BACKES, C. Níveis crescentes de saturação por bases e desenvolvimento inicial de teca em um Latossolo de textura média. Ciência Florestal, v. 22, n. 4, p. 693-702, 2012.

FEITOSA, D. G. et al. Crescimento de mudas de gonçalo-alves (Astronium fraxinifolium) sob diferentes fontes e doses de nitrogênio. Revista Árvore, v. 35, n. 3, p. 401-411, 2011.

FREEDMAN, B. Environmental ecology: The ecological effects of pollution, disturbance and other stresses. San Diego: ACADEMIC PRESS, 1995.

LOCATELLI, M.; MACÊDO, R. S.; VIEIRA, A. H. Avaliação de altura e diâmetro de mudas de cedro rosa (Cedrela odorata L.) submetidas a diferentes deficiências nutricionais. Revista Brasileira de Biociências, v. 5, supl. 2, p. 645647, 2007.

MAGALHÃES, J. R. Diagnose de desordens nutricionais em hortaliças. Brasília: EMBRAPA-CNPH, 1998. 64 p.

MALAVOLTA, E. Elementos de nutrição mineral de plantas. São Paulo: CERES, 1980. $251 \mathrm{p}$.

MALAVOLTA, E.; VITTI, G. C.; OLIVEIRA, S. A. Avaliação do estado nutricional das plantas: princípios e aplicações. 2. ed. Piracicaba: POTAFOS, 1997. 319 p.

MARQUES, T. C. L. L. S. M. et al. Crescimento inicial do paricá (Schizolobium amazonicum) sob omissão de nutrientes e de sódio em solução nutritiva. Cerne, v. 10, n. 2, p. 184-195, 2004.

MARSCHNER, H. Mineral nutrition of higler plants. 2. ed. New York: ACADEMIC PRESS, $1995.889 \mathrm{p}$.

MATHEUS, M. T. et al. Sintomas de deficiência nutricional em plantas de jatobá. Revista Científica Eletrônica de Engenharia
Florestal, v. 17, n. 1, p. 89-97, 2011.

MENDONÇA, A. V. R. et al. Exigências nutricionais de Myracrodruon urundeuva Fr. All (aroeira do sertão). Cerne, v. 5, n. 2, p. 65-75, 1999.

MORETTI, B. S. et al. Crescimento e nutrição mineral de mudas de cedro australiano (Toona ciliata) sob omissão de nutrientes. Cerne, v. 17, n. 4, p. 453-463, 2011.

PEDROSO, A. N. V. Poluentes atmosféricos e plantas bioindicadoras. 2007. Disponível em: $<$

http://www.biodiversidade.pgibt.ibot.sp.gov.br/ Web/pdf/Poluentes_Atmosfericos_\&_Plantas_ Bioindicadoras_Andrea_N_V_Pedroso.pdf >. Acesso em: 15 dez. 2014.

RAIJ, B. V. Fertilidade do solo e adubação. Piracicaba: POTAFOS, 1991. 343 p.

RIBEIRO, F. A. et al. Efeitos da adubação de plantio sobre o estabelecimento de mudas de Tectona grandis L.f. (teca). Revista Científica Eletrônica de Engenharia Florestal, ano. 4, n. 7, [s.p.], 2006.

SARCINELLI, T. S. et al. Sintomas de deficiência nutricional em mudas de Acacia holosericea em resposta à omissão de macronutrientes. Revista Árvore, v. 28, n. 2, p. 173-181, 2004.

SARRUGE, J. R. Soluções nutritivas. Summa Phytopathologica, v. 1, n. 3, p. 231-233, 1975.

SILVA, F. de A. S.; AZEVEDO, C. A. V. de. Versão do programa computacional Assistat para o sistema operacional Windows. Revista Brasileira de Produtos Agroindustriais, v. 4, n. 1, p. 71-78, 2002.

SILVA, E. B.; GONÇALVES, N. P.; PINHO, P. J. Limitações nutricionais para crescimento de mudas de umbuzeiro em Latossolo Vermelho distrófico no Norte de Minas. Acta 
Scientiarum. Agronomy, v. 27, n. 1, p. 55-59, 2005.

SILVA, A. R. M. et al. Efeitos de doses crescentes de calcário na produção de mudas de sumaúma (Ceiba pentandra L. Gaertn). Floresta, v. 38, n. 2, p. 295-302, 2008.

SILVA, R. C. B.; SCARAMUZZA, W. L. M. P.; SCARAMUZZA, J. F. Sintomas de deficiências nutricionais e matéria seca em plantas de nim, cultivadas em solução nutritiva. Cerne, v. 17, n. 1, p. 17-22, 2011.

SORREANO, M. C. M. et al. Deficiência de macronutrientes em mudas de sangra d'água (Croton urucurana, Baill.). Cerne, v. 17, n. 3, p. 347-352, 2011.

SOUZA, C. A. S. et al. Exigências nutricionais e crescimento de plantas de mogno (Swietenia macrophylla King.). Acta Amazônica, v. 40, n. 3, p. 515-522, 2010.

TAIZ, T.; ZEIGER, E. Fisiologia vegetal. 3. ed. Porto Alegre: ARTMED, 2004. 719 p.

VALERI, S. V. et al. Efeitos da omissão de nutrientes em plantas de Caesalpinia echinata. Cerne, v. 20, n. 1, p. 73-80, 2014.

VENTURIN, N. et al. Adubação mineral da candeia (Eremanthus erythropappus (DC.) McLeish). Floresta, v. 35, n. 2, p. 211-219, 2005.

VIEIRA, H.; CHAVES, L. H. G.; VIÉGAS, R. A. Crescimento inicial de moringa (Moringa oleífera Lam) sob omissão de nutrientes. Caatinga, v. 21, n. 4, p. 51-56, 2008.

VIEIRA, C. R.; WEBER, O. L. S.; SCARAMUZZA, J. F. Descrição de sintomas visuais em função das deficiências de macronutrientes em mudas de cerejeira (Amburana acreana). Floresta, v. 41, n. 4, p. 789-796, 2011.
WALLAU, R. et al. Sintomas de deficiências nutricionais em mudas de mogno cultivadas em solução nutritiva. Cerne, v. 14, n. 4, p. 304-310, 2008.

ZHANG, F.; NIU, J.; ZHANG, W.; CHEN, X.; LI, C.; YUAN, L.; XIE, J. Potassium nutrition of crops under varied regimes of nitrogen supply. Plant and Soil, v. 335, p. 21-34, 2010. 